\title{
El Aprendizaje Servicio como un proceso de innovación y generación de talento Learning and Service as a process of innovation and generation of talent
}

\author{
Isabel Acero Fraile ${ }^{1}$, Estrella Bernal Cuenca ${ }^{1}$, Gemma Larramona Ballarín ${ }^{2}$, Ana Katarina Pessoa De Oliveira ${ }^{3}$ \\ iacero@unizar.es, bercue@unizar.es,gemmalar@unizar.es, apessoa@unizar.es
}

\author{
${ }^{1}$ Departamento de Dirección y Organización de \\ Empresas \\ Facultad de Economía y Empresa \\ Universidad de Zaragoza \\ Zaragoza, España
}

\author{
${ }^{2}$ Departamento de Análisis Económico \\ Facultad de Economía y Empresa \\ Universidad de Zaragoza \\ Zaragoza, España
}

\author{
${ }^{2}$ Departamento de Contabilidad y \\ Finanzas \\ Facultad de Economía y Empresa \\ Universidad de Zaragoza \\ Zaragoza, España
}

\begin{abstract}
Resumen- En el nuevo contexto social en el que las nuevas tecnologías pueden limitar la creatividad de las personas, surge la práctica de la metodología "Aprendizaje y Servicio (ApS)" que rompe las fronteras de las aulas y sale del ambiente estrictamente universitario, haciendo que el intercambio de conocimientos en los entornos personalizados de la sociedad sean espacios para crear y crecer de manera multi, inter y transdisciplinar tanto para el alumnado como para los usuarios de las entidades privadas sin ánimo de lucro. El objetivo es presentar una propuesta de metodología ApS desarrollada en la Facultad de Economía y Empresa de la Universidad de Zaragoza como un proceso de cambio tendente a la generación de innovación y desarrollo del talento en el alumnado basado en competencias técnicas, personales e intrapersonales, así como de generación de valor en el sistema socioeconómico.
\end{abstract}

Palabras clave: Aprendizaje y servicio. Proceso de innovación. Generación de talento.

Abstract- In the new social context in which new technologies can limit the creativity of people, the practice of the "Learning and Service (ApS)" methodology breaks down the boundaries of the classroom and leaves the strictly university environment, making the knowledge exchange in the personalized environments of society spaces to create and grow in a multi, inter and transdisciplinary way for both students and users of private non-profit entities. The objective is to present the proposal ApS methodology developed at Faculty of Business and Economy at University of Zaragoza as a process of change focusing on the generation of innovation and talent development in students based on technical, personal and intrapersonal skills, as well as generation of value in the socioeconomic system.

Keywords: Learning and service. Innovation process. Generation of talent.

\section{INTRODUCCIÓN}

En los tiempos en que la cibernética puede llegar a solucionar la mayor parte de las tareas que no requieren creatividad en las empresas y en la sociedad en general, el desarrollo de las competencias vinculadas a dicha creatividad es clave para la empleabilidad de nuestros futuros graduados. La metodología ApS, más allá del evidente valor social que genera, puede sostener potentes procesos de creatividad del alumnado susceptibles de plasmarse en modos innovadores de comunicar conocimientos para solucionar situaciones prácticas y reales que se dan en las organizaciones a las que se dirigen.

El objetivo de esta práctica que realizan los alumnos es aprender haciendo un servicio a la comunidad. En el aprendizaje-servicio, el alumnado identifica en su entorno una o varias organizaciones sin ánimo de lucro necesitadas de unos conocimientos que él dispone por su formación en economía y empresa; se compromete a diseñar un programa que sea capaz de dar respuesta a dichas necesidades y adaptar los contenidos al colectivo en el que se vaya a desarrollar. Una vez diseñado el programa deberá exponerlo en el centro con el que ha estado en contacto y en algún otro centro con una realidad social similar.

De esta forma se desarrolla un proyecto solidario basado en la transferencia de conocimiento que pone en juego habilidades, actitudes y valores. Además el alumno desarrollará una habilidad fundamental para la vida profesional como es la comunicación, tanto verbal como no verbal y el saber adaptarse en cada momento a las necesidades del público que tiene delante.

\section{CONTEXTO}

Este proyecto parte de la iniciativa de varios profesores de la Facultad de Economía y Empresa de la Universidad de Zaragoza motivados e interesados en la metodología ApS. El objetivo es desarrollar una actividad de Aprendizaje-Servicio en la que los estudiantes cambien su rol como alumnos y se conviertan en docentes con el valor añadido de que la formación que impartan va a ir dirigida a colectivos de organizaciones sin ánimo de lucro por lo que también van a desarrollar un rol de carácter social.

A continuación se reflexiona sobre la importancia de metodologías como el ApS y su efecto en el aprendizaje y los niveles de atención así como el fomento de la innovación y excelencia.

A. Entornos nuevos de aprendizaje y niveles de atención 
Diversos estudios de neurociencias corroboran el hecho de que los entornos nuevos, aumentan los niveles de atención, usando y reforzando la plasticidad del cerebro.

Un elemento fundamental de nuestra metodología ApS son precisamente los entornos nuevos a los que se enfrenta el alumnado, donde han de realizar su labor didáctica, en nuestro caso, organizaciones sin ánimo de lucro. Estas organizaciones incluyen un amplio abanico de entidades aunque no todas ellas son objeto de una práctica de este tipo. Estas organizaciones tienen que cumplir varios requisitos. El primero y fundamental para que la entidad pueda ser considerada en el proyecto es que la formación que el alumno vaya a hacer no sustituya un trabajo que se realizaba ya previamente o un servicio que la entidad tuviera pensado realizar acudiendo al mercado para contratarlo. La formación que realice el alumno no debe sustituir el trabajo de ninguna persona presente ni futura.

Los siguientes requisitos que debe cumplir se podrían enmarcar dentro de propósitos más generales y son tres: que las entidades sean socialmente responsables; ecológicamente sostenibles; y que además tengan un impacto social dentro del entorno. Esto restringe un poco el tipo de entidades con las que trabajar, aunque el abanico sigue siendo muy amplio. Este proyecto se puede desarrollar en: centros de día de la tercera edad, asociaciones de barrio, centros educativos de zonas económicamente deprimidas, asociaciones de inmigrantes y un largo etcétera que tiene que aunar expectativas de oferentes y demandantes de la formación. Siguiendo este esquema entendemos que ambas partes son lo suficientemente flexibles para poder ajustar sus expectativas y así detallar un proyecto educativo en el que el alumno (oferente) se sienta cómodo y a la vez satisfaga las necesidades de la entidad (demandante). Una vez concretados los contenidos se adecúan al nivel requerido en cada una de las entidades, así pues el alumno debe adaptar sus conocimientos al público al que se dirige.

El hecho de enfrentarse a una situación nueva tanto en lo concerniente al espacio físico como cultural de la organización anfitriona del alumnado, y a su propia posición de docente como contraposición al rol habitual de alumnado, hace que, en términos de programación neuro lingüística, el modelado de la realidad habitual quede descolocado. Ello implica la búsqueda de modelos alternativos de observar y comprender la realidad, que lleva al desarrollo de nuevas sinapsis cerebrales y desarrollo de la consiguiente neuroplasticidad.

De acuerdo con Ben-Soussan, Glikcsohn y BerkovichOhana (2017), Curry (2017) y Mrazek, Mooneyham, Mrazek y Schooler (2016), el éxito de la resolución de esta situación nueva, depende en gran medida del grado de atención que se desarrolle en el momento presente, el cual está precisamente relacionado con esta capacidad de mirar a la realidad desde nuevas perspectivas.

\section{B. Una relectura del Proceso ApS desde la Teoría U} como generador de innovación y excelencia

Esta mirada desde nuevas perspectivas es el primer paso de lo que se denomina como proceso $U$ de innovación y de cambio según la Teoría U (Sharmer, 2016). Significa pasar del nivel habitual de descarga de los patrones aprendidos de percepción de la realidad, proyectando los propios mapas mentales, a un nivel de un mayor análisis donde hay una percepción de la realidad más observadora a nivel de hechos, que no juzga perspectivas diferentes a la propia habitual, sino que las observa y analiza con curiosidad para falsar la propia percepción. Es lo que la teoría U llama nivel de “mente abierta”.

Si el alumnado ha de enfrentarse a un entorno nuevo organizacional y cultural, con personas diferentes y con un rol totalmente distinto al que está acostumbrado a desarrollar, y se espera tener éxito en la responsabilidad que se le ha asignado de transmitir una serie de conocimientos, será necesario que practique esta apertura de mente, principalmente al inicio de su relación con la entidad cuando tenga que realizar un análisis de la organización para detectar sus necesidades. Será necesario, por tanto, pararse y observar la organización, separarse de su visión habitual de los conocimientos de las materias implicadas como estudiante de las mismas, y enfocarlas de un nuevo modo para ayudar a quienes pretende transmitirlas, a resolver problemas concretos a los que se enfrentan.

Es aquí, en este tratar de ayudar a resolver problemas concretos, donde entra en juego una segunda fase de nuestra metodología ApS, orientada a la captación de necesidades en la organización y elaboración de un proyecto educativo. Esta captación de necesidades implica una capacidad de observación más profunda y amplia que la mera mente abierta, sobre todo cuando el estudiante se enfrente a organizaciones que tal vez trabajen con colectivos desfavorecidos socialmente, o simplemente con niveles culturales básicos. Dicha capacidad es lo que la Teoría U llama corazón abierto, e implica la capacidad de empatía y observación del sistema desde los valores del otro, desde el ser capaz de adoptar su posición integral como ser humano, y captar sus valores así como sus necesidades reales de aprendizaje. Es lo que de modo natural sucede cuando estamos absolutamente atentos al otro/a en nuestra interacción con él o ella, lo que sucede cuando un buen docente conecta con su alumnado más allá del mero discurso intelectual y percibe sus necesidades de aprendizaje a nivel humano integral. Es un simple desarrollo de relación humana de igual a igual, que siendo absolutamente natural en los seres humanos (Maturana, 1975), queda marginado en muchas ocasiones en la distribución de roles que se da entre quien enseña como poseedor del conocimiento y quien aprende como demandante del mismo, y donde el proceso de aprendizaje parece darse como una suerte de transacción en la que alguien da conocimiento a cambio de algo.

Es la atención plena en el momento presente lo que facilita tanto la mente abierta, como el corazón abierto, como lo que la Teoría U llama voluntad abierta, aquella que permite dejar ir viejos modos de comportamiento y que emerja lo nuevo que hay en la situación que se nos presenta. Una gran atención aquí y ahora, a lo que el estudiante tiene como bagaje personal, único, individual e irrepetible, y que surge de modo espontáneo cuando hay que resolver situaciones nuevas y complejas, cuando parece que en nuestro acervo de conocimientos intelectuales no hay lo necesario para abordar 
una situación, es cuando surge nuestra fuente original de creatividad y talento (Pelser, Bosch y Schurink, 2016 y Staub, 2016), que a su vez da una relectura al conocimiento con el que contamos.

En el contexto de la Teoría U, que es fruto de un trabajo empírico de más de dos décadas observando procesos sociales y organizacionales, Sharmer (2013) explica que el proceso creativo desde la mente abierta, corazón abierto y voluntad abierta, se potencia de modo extraordinario cuando tiene lugar en la co-creación y co-inspiración del diálogo profundo y generativo. Este diálogo sucede en ese punto de atención plena al otro en el aquí y ahora, donde se da una escucha sin juicios y atenta a las necesidades, perspectiva y valores del otro.

\section{Aprendizaje-Servicio}

Tal y como define en su web la Red Española de Aprendizaje-Servicio, el ApS es "Aprender haciendo un Servicio a la comunidad” (https://aprendizajeservicio.net/quees-el-aps/). En el aprendizaje-servicio, el alumnado cambia su rol como estudiantes y su prioridad es identificar una necesidad en algún colectivo que pueda mejorarse a través de un proyecto solidario (en nuestro caso, proyecto educativo). Es una práctica educativa en la que los estudiantes aprenden mientras actúan sobre necesidades reales y realizan por tanto una labor social.

Tal y como señala la Red Española de AprendizajeServicio, a través de esta metodología se fomentan por un lado Competencias básicas ya que se aplican diversas competencias del currículo, con énfasis en la competencia social y ciudadana y en la iniciativa y autonomía personal. Asimismo se promueven Valores y actitudes prosociales, estimulando el esfuerzo, la responsabilidad y el compromiso solidario. Y finalmente, pero no por ello menos importante, se desarrollan Habilidades para la vida, fortaleciendo las destrezas psicosociales y la capacidad de participar en la vida social de manera positiva.

El Aprendizaje Servicio es, por tanto, una vía de acercar la Universidad a la sociedad de hoy en día ya que tal y como ponen de manifiesto Rubio, Prats y Gómez (2013): "Propuestas formativas como el aprendizaje servicio muestran que es posible una universidad que encuentra el equilibrio entre el rigor científico y el compromiso social, entre la excelencia académica y la equidad. En definitiva, el ApS permite concretar la misión de la responsabilidad social y materializar un modelo de universidad en permanente relación y comprometida con la sociedad de la que forma parte”.

Hay que tener en cuenta que no hay que confundir Aprendizaje-Servicio con voluntariado. Tal y como apunta Martínez (2008), las actividades de ApS deben tener, por un lado, un componente académico (tienen que suponer un aprendizaje académico en los estudiantes), y por otra parte, deben favorecer la mejora de la calidad de vida e inclusión social del colectivo al que se dirigen.

\section{DESCRIPCIÓN}

La metodología ApS, dentro del proyecto que se realizará con los alumnos de la Facultad y dado el carácter pionero de esta iniciativa en nuestro Centro, se centra principalmente en el aspecto formador del alumno, pudiéndose extender a cualquier otro ámbito en el que el propio alumno tiene capacidades. La actividad se articula en la modalidad de "prácticas en empresa” aunque en este caso la "empresa” en la que se realizarán las prácticas no será una empresa como tal sino una organización sin ánimo de lucro, siendo éste uno de los pilares fundamentales de la metodología ApS.

Los alumnos de la Facultad de Economía y Empresa de Zaragoza tienen la posibilidad de realizar prácticas en empresa tanto de forma curricular (dentro de los créditos que forman su titulación) como de forma extracurricular (como un complemento formativo a su titulación). Con este proyecto que se va a implementar en el curso 2017-2018 se incluye la posibilidad de que los estudiantes que así lo deseen puedan realizar sus prácticas en empresa en la línea de trabajo del Aprendizaje Servicio. Estos estudiantes, realizarán prácticas en empresa pero la organización en la que desarrollarán su trabajo será una entidad sin ánimo de lucro (asociación de barrio, asociación de inmigrantes, etc.) y su labor en dicha organización tendrá un componente formativo ya que los alumnos deberán llevar a cabo un proyecto educativo para implementar en la organización de destino, a través del cual puedan transmitir su formación en aspectos económicoempresariales y adaptarla a las necesidades de la entidad en la que realizarán sus prácticas.

Las prácticas a realizar por el alumno tendrán una duración de 120 horas totales distribuidas de la siguiente forma:

1) Las primeras 8 horas se repartirán en cuatro sesiones donde se complementará la formación del alumno con formación en tres temas muy concretos. Por un lado, formación sobre la propia metodología ApS que es novedosa en nuestra Facultad ya que es necesario que los estudiantes conozcan su esencia y la forma de llevarla a cabo. Por otro lado, formación en habilidades comunicativas, que les serán de gran utilidad a los estudiantes para entrar en contacto con la entidad en la que van a desarrollar sus prácticas. Y finalmente, se les ofrecerá formación sobre realización de entrevistas para poder captar de una forma rigurosa las necesidades (formativas) de la entidad sin ánimo de lucro en la que se desarrollará el proyecto cada estudiante. Estas necesidades formativas estarán relacionadas con contenidos que pueden ser de análisis económico, organización de empresas, finanzas, contabilidad y/o marketing, es decir, la formación de base que reciben los estudiantes de la Facultad.

2) A continuación el alumno acudirá diariamente a la entidad asignada para conocer la organización in situ. Allí desarrollará una memoria donde explicará el organigrama de la entidad, identificará las personas responsables en la toma de decisiones, el tipo de actividad que desarrollan, las características socioeconómicas de los usuarios de las actividades de la entidad..., es decir, deberá realizar una labor de conocimiento profundo de la entidad. Además, completará esta labor con la realización de entrevistas con el objetivo de analizar la necesidad/demanda de formación en temas de economía y empresa que tenga la entidad, estudiando también 
el perfil de las personas que recibirán dicha formación. Esta parte tendrá una duración de unas 30h aproximadamente y será la base para la elaboración del Proyecto Educativo que debe realizar el estudiante en la entidad y que constituye la siguiente etapa que se describe a continuación.

3) Unas 45 horas aproximadamente se destinarán a la realización del Proyecto Educativo donde se trabajarán los contenidos apropiados para la entidad y con un nivel de profundidad adecuado para el público objetivo que forma esa entidad. Con la información obtenida en la fase anterior sobre las necesidades/demanda formativa que tenga la entidad, el estudiante elaborará un Proyecto Educativo en el que se describan las charlas, actividades, talleres y demás recursos educativos que utilizará el alumno. Se propone el siguiente ejemplo con el objetivo de facilitar la comprensión del lector y clarificar las ideas. Si el alumno ha detectado, por ejemplo, que es necesario formar a los diferentes usuarios y personal de la organización en la que realiza sus prácticas en temas de carácter financiero, desarrollará todo un programa formativo en el que detalle los conceptos a trasmitir, el modo de hacerlo, los recursos a utilizar. Para ello, utilizará la formación recibida a lo largo de su titulación pero la tendrá que adaptar al público al que se dirige adaptando tanto el nivel cómo la forma de transmitirlo. Una vez elaborado el programa de formación, el alumno realizará una primera presentación en el centro en el que ha desarrollado sus prácticas (asociación de vecinos, asociación de inmigrantes, etc.) a la que seguirán otras 4 ó 5 exposiciones más sobre el mismo tema en centros similares hasta hacer un total de unas 21 horas. El hecho de "repetir" su presentación en varias ocasiones permite que el alumno vaya aprendiendo de sus errores ya que recibirá un feedback después de cada sesión.

4) Finalmente, la actividad contará con su evaluación correspondiente. La evaluación será por pares, de la institución, del tutor académico y del propio alumno (autoevaluación) y al conjunto de ella se destinan 16 horas. La evaluación será tanto intermedia, conforme el estudiante realiza las presentaciones, como evaluación final, al acabar todo el proceso. Además, los estudiantes tienen asignadas unas horas para acompañar en las conferencias/charlas a otros compañeros con el objetivo de que se complemente su informe de evaluación final que contribuirá a destacar aspectos a mejorar o fortalecer. Esto además, les permitirá obtener un feedback después de cada sesión que ayudará a mejorar las siguientes exposiciones. Para la realización de las evaluaciones se utilizará un sistema de rúbricas.

En la siguiente figura (Figura 1) se recoge el esquema de trabajo a desarrollar.

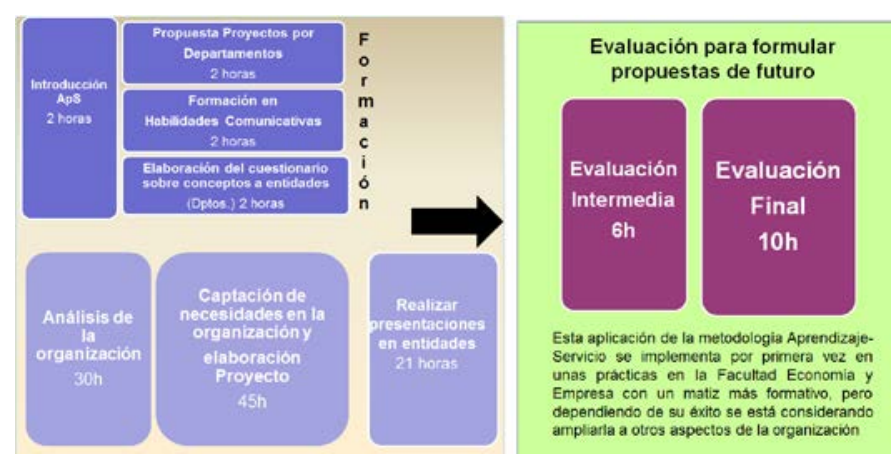

Figura 1 - Metodología de Aprendizaje y Servicio en la Facultad de Economía y Empresa de Zaragoza

Más allá de los beneficios que la metodología ApS tiene en el aprendizaje de las propias materias en que el alumnado se involucra, planteamos una serie de relaciones causa efecto teóricas que nos llevarán a sostener el que esta metodología desarrolla el talento natural del alumnado, así como procesos de innovación social en los que las competencias del talento natural son fuentes de excelencia en el desempeño profesional, con la consecuente generación de valor añadido para el sistema socioeconómico.

Con esa metodología se espera alcanzar el principal objetivo del proyecto ApS: que el alumnado sea capaz de transferir y captar conocimientos en entornos personalizados de manera multi, inter y transdisciplinar, desarrollando las competencias de cada área de la enseñanza en el ámbito de economía y empresa, por ejemplo, algunas como:

- Comprender las estrategias micro y macroeconómicas y sus implicaciones en la gestión de una entidad;

- Identificar situaciones de optimización de recursos y costes;

- Valorar la situación y la evolución previsible de empresas y organizaciones, tomar decisiones y extraer el conocimiento relevante;

- Emitir informes de asesoramiento sobe situaciones concretas de mercados, sectores, organizaciones, empresas y sus áreas funcionales.

- Identificar, interpretar y evaluar la información financiera y contable de las empresas e instituciones para identificar las fortalezas y debilidades, así como para asesorar desde un enfoque técnico, financiero y contable en la toma de decisiones;

- Analizar, valorar y tomar decisiones de marketing en relación a sectores de actividad con características específicas.

\section{Resultados}

Dado que se trata de una iniciativa pionera en el ámbito de la Facultad de Economía y Empresa de Zaragoza, todavía no se tienen resultados derivados de su implantación puesto que se espera poner en marcha durante el próximo curso 20172018. 
No obstante, sí que se han establecido los criterios con los que se evaluará la labor realizada por los estudiantes y que constará tanto de una evaluación intermedia como de una evaluación final. Para ello, se aplicará un sistema de evaluación por pares y autoevaluación, en el que intervendrán tanto miembros de la institución donde el alumno ha desempeñado su labor, como el tutor académico, así como el propio alumno. Además, a las sesiones expositivas también acudirán otros compañeros del estudiante con el objetivo de aportarle feedback sobre su presentación y poder realizar las mejoras que se estimen oportunas de cara a las siguientes exposiciones.

\section{CONCLUSIONES}

Teniendo en cuenta el proyecto presentado en este trabajo, podemos decir que la metodología $\mathrm{ApS}$, que enfrenta al alumnado a entornos nuevos con colectivos con necesidades especiales de aprendizaje le ofrece la posibilidad de conectar con su potencial profundo de talento, y desde este punto de consciencia de sí mismo y del entorno nuevo, involucrarse en relaciones humanas con una comunicación desde un diálogo generativo y atento desde el que co-crear e innovar.

En nuestra metodología ApS, la labor de realizar las presentaciones de conceptos en entidades sin ánimo de lucro, es una oportunidad única para el desarrollo de las competencias desde este talento, pero competencias no sólo técnicas, sino también interpersonales e intrapersonales, que constituyen la base sobre la que se compone un desempeño profesional excelente (Goleman et al., 2013), con capacidad de generación de valor añadido no sólo a nivel económico (Armstrong, 2014) sino también social (Sharmer, 2013).

Es importante resaltar que este desarrollo del talento potencial del alumnado, requiere del acompañamiento de profesorado universitario que desee involucrarse también en este proceso de cambio y búsqueda del propio potencial y talento. Todo ello es fuente inagotable de nueva creatividad y co-generación de soluciones para los acuciantes problemas el sistema socioeconómico.

Finalmente señalar que esta experiencia se podría replicar en otros ámbitos y contextos universitarios y con diferentes organizaciones no lucrativas, por lo que la sostenibilidad del proyecto así como su transferibilidad son ilimitadas. Asimismo, como el proceso de aprendizaje debe ser continuo, iremos mejorando el proyecto aquí presentado conforme se vaya desarrollando.

\section{AGRADECIMIENTOS}

Agradecemos al proyecto "Laboratorio de Economía Social LAB_ES” de la Facultad de Economía y Empresa la financiación facilitada a través del Convenio con Zaragoza Dinámica, entidad perteneciente al Ayuntamiento de
Zaragoza, para la inscripción a este Congreso y el apoyo en el desarrollo de la actividad

\section{REFERENCIAS}

Armstrong, M. \& Taylor, S. (2014). Armstrong's Handbook of Human Resources Management Practice. London: Kogan Page.

Ben-Soussan, T.D., Glicksohn, J. \& Berkovich-Ohana, A. (2017). Attentional effort, mindfulness, and altered states of consciousness experiences following quadrato motor training. Mindfulness, 8 (1) (FEB), 59-67.

Curry, C. (2017). Stress-proof brain: Master your emotional response to stress using mindfulness and neuroplasticity. Library Journal. 142 (2) (FEB 1), 95-113.

Goleman, D., Boyatzis, R. \& Mckee,A. (2013): Primal Leadership. Harvard Business School Publishing. Boston, Massachusetts.

Martínez, M. (2008) (coord.). Aprendizaje Servicio y responsabilidad social de las universidades. Educación Universitaria, Octaedro / ICE - UB.

Maturana, H. (1975). The organisation of the living: a theory of the living organisation. Journal of Man Machine Studies, 7, 313-332.

Mrazek, M.D., Mooneyham, B.W., Mrazek, K.L., \& Schooler, J.W. (2016). Pushing the limits: Cognitive, affective, and neural plasticity revealed by an intensive multifaceted intervention. Frontiers in Human Neuroscience 10 (MAR 18), 117.

Pelser, H.J., Bosch, A., \& Schurink, W. (2016). An organisational coherence model to maintain employee contributions during organisational crises. SA Journal of Human Resource Management/SA Tydskrif vir Menslikehulpbronbestuur, 14(1), a725. http://dx.doi. org/10.4102/sajhrm. v14i1.725

Rubio, L., Prats, E., \& Gómez, L. (2013) (coord.). Universidad y sociedad. Experiencias de aprendizaje servicio en la universidad. Institut de Ciències de l’Educació.

Sharmer, O. \& Kaufer, K. (2013). Leading From the Emerging Future: From Ego-system to Eco-system Economies . San Francisco, CA: Berrett-Koehler Publishers.

Sharmer, O. (2016). Theory U: Leading from the Future as it Emerges 2nd Edition. San Francisco, CA: BerrettKoehler Publishers

Staub, M. E. (2016). The other side of the brain the politics of split-brain research in the 1970s-1980s. History of Psychology 19 (4) (NOV), 259-73. 